\title{
Non-spherical Outflows in Massive Binary Systems: Circumbinary Disks?
}

\author{
Gloria Koenigsberger, Edmundo Moreno, and Jorge Cantó \\ Instituto de Astronomía, UNAM
}

\begin{abstract}
The trajectories of wind particles emitted from the surface of a massive star in a close binary system are analyzed. Within a radius 1000 times the separation of the two stars, a significant fraction of the particle trajectories are found to cross the orbital plane, leading to the formation of a large-scale, outflowing circumbinary disk-like structure. The shocks which arise due to the collision of particles which are within crossing streamlines are expected to produce selected regions within the wind, particularly in the orbital plane, in which a higher degree of ionization prevails than in the wind in general. X-ray emission might also be expected from these regions. Such a model is suggested to be applicable to the erupting WR/LBV binary system HD 5980 and other binary LBV systems, during phases when wind velocities are slow.
\end{abstract}

\section{Introduction}

The interaction between stars in close binary systems propitiates the presence of non-spherically symmetric mass outflows. Wind-wind collisions and stellar rotation are mechanisms capable of producing departures from spherically symmetric mass loss, as well as the mechanisms assumed to be active in single stars particularly if magnetic fields are present. Many of these mechanisms are described in other papers presented in this Colloquium and, in Drissen et al. (1992). In this paper we turn our attention to the effects that the gravitational and centrifugal forces produce on the emerging wind of one of the stars in the system, and propose that under certain conditions in massive binary systems, a significant amount of the ejected material accumulates in the orbital plane, producing a circumbinary disk-like structure. The conditions most suited to produce this outflow morphology occur in the LBV phase of the star's evolution, when the wind velocity is slow.

Concentrations to the orbital plane of outflowing mass in binary systems has been predicted for low-mass systems in which a common envelope phase occurs (see review by Iben and Livio 1993). Two- and three-dimensional calculations performed by Bodenheimer and Tamm (1984), Livio and Soker (1988), Taam and Bodenheimer $(1989,1991)$ and Terman et al. (1994) demonstrate that the ejection of envelope material takes place preferentially in the orbital plane in these systems. Although similar to a stellar wind in that it is not corrotating with the binary system, a common envelope, however, differs significantly in its radial velocity and density structure from the stellar 
winds in massive stars. Hence, the results for the mass-outflow distribution in common envelope phases are not applicable to the massive star case. Thus, calculations need to be performed for conditions prevailing in massive stars.

Mechanisms for producing a concentration of gas in the orbital plane are of interest, because, among other reasons, such a density contrast is believed to be responsible for the bipolar morphologies observed in $\mathrm{H}$ II regions surrounding LBV systems such as Eta Carinae, AG Car and other LBV's (Frank 1997; Garcia-Segura et al.1997). Eta Car is now strongly suspected to be a binary system (Damineli et al. 1997; Lamers et al. 1998), and its surrounding H II region was formed at the time of the 1850 Great Eruption. Furthermore, the presence of a wind confined to a disk is suggested for other massive systems, in particular WR 140 (White and Becker, 1995).

\section{Formation of a Circumbinary Disk-Like structure in Massive Binaries}

In order to analyze the morphology of the outflow from a massive binary system, the trajectories of individual particles within the system were calculated by solving the equation of motion in the frame of reference which is rotating with the binary. We performed a numerical experiment for the case of a $85 M_{\odot}+30 M_{\odot}$ (= star A + star B) system, with a circular orbit and separation of $70 R_{\odot}$. The assumed stellar radii are $48 R_{\odot}$ and $15 R_{\odot}$, respectively, and the system is assumed to be in corotation. The wind of star A is assumed to have a velocity of $1000 \mathrm{~km} \mathrm{~s}^{-1}$. The wind of star B is ignored for the present calculation. Particles were released radially from the surface of star $\mathrm{A}$ at spatial intervals of 1 degree in both the $\theta$ and $\phi$ coordinates. The polar angle $\theta$ is measured from the north pole of star A and the azimuthal angle $\phi$ is measured from the line joining the two stars in the direction of the orbital motion. Particles which reached a sphere with radius 1000 times the orbital separation whose origin is the center of mass, were assumed to escape the system and their motion was not followed beyond this point. Particles which collide with Star B are assumed to be trapped and disappear from the calculation.

In Figure 1 we illustrate the velocity field of the particles which cross the orbital plane ( $\mathrm{x}-\mathrm{y}$ plane), measured with respect to the inertial reference frame whose origin is the center of mass of the system. This is the velocity field in the observer's frame of reference. The length of the arrows indicates the magnitude of the velocity component in the $x-y$ plane. These velocities range from close to zero (i.e., the motion is mainly perpendicular to the $x-y$ plane) to more than $500 \mathrm{~km} \mathrm{~s}^{-1}$. Star A is located at $(-\mu, 0)$ while Star B is at $(1-\mu, 0)$, where $\mu=M_{B} /\left(M_{A}+M_{B}\right)$. The values of $\mathrm{x}$ and $\mathrm{y}$ are in units of the orbital separation. Thus, one finds that a large number of particles intersect the orbital plane at distances of $280 R_{\odot}$ or more. 


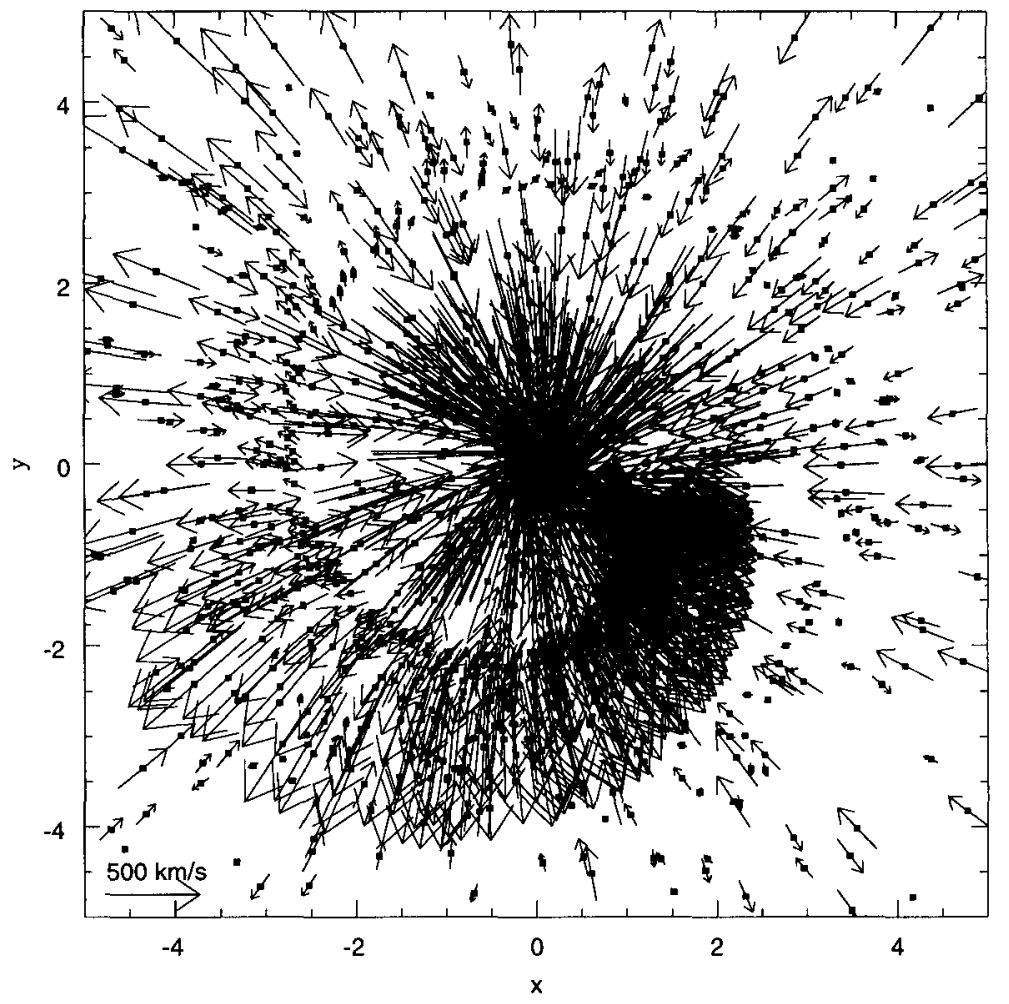

Fig. 1. Distribution of particles which cross the orbital plane. The length of the arrows corresponds to the velocity component in the $x-y$ plane. Orbital motion is counterclockwise. Star A lies nearly at the center of the figure. The $\mathrm{x}$ and $\mathrm{y}$ axes are in units of the orbital separation.

The particles which are deflected towards the orbital plane are found to emerge from specific regions of the stellar surface. For the parameters listed above and the stated assumptions, the particles which tend to have trajectories which take them towards the orbital plane are those emerging from polar angles $\theta>53$ degrees. For angles smaller than this, the particles escape the system. Furthermore, the majority of the particles whose trajectories take them across the orbital plane emerged from azimuthal angles between -20 and +70 degrees; that is, from the portion of the star facing the companion. For a given set of binary star parameters, the slower the initial particle ve- 
locity, the larger is the number of particles which are deflected towards the orbital plane.

It is important to note that the results presented in Figure 1 correspond to particles released only in one of the hemispheres of star A. Since the forces acting in the system are the same in the northern and southern hemispheres and in both cases particles converge towards the orbital plane, a region of shock formation is expected to arise. The strength of the shocks will be proportional to the velocity components perpendicular to the $\mathrm{x}-\mathrm{y}$ plane. In addition, shocks are expected to be formed above and below the orbital plane, as particle trajectories cross. Depending on the velocities with which these shocks occur, emission lines from a variety of ionization species are expected, most of which with higher degrees of ionization than the general wind. One would also expect soft X-ray emission produced in this process. Before quantifying these effects, however, the collision of particles has to be incorporated into the calculation. Three additional effects need to be considered as well: radiation pressure; the eccentricity of the orbit; and the possibility that the stellar surface from which the wind is assumed to be emerging is not spherically symmetric to begin with (Fliegner and Langer 1995; Cassinelli, Ignace and Bjorkman 1995).

\section{A Circumbinary Disk in HD 5980?}

The close binary system $(P=19.3$ days $)$ HD 5980 in the Small Magellanic Cloud, has an eccentric ( $e=0.3$ ) orbit and a significant orbital inclination $(i>0.87)$. Classified as a WN4 + O7I: Wolf-Rayet system in the late 1970's, its wind velocities were near $3000 \mathrm{~km} \mathrm{~s}^{-1}$. Over the next few years, the wind velocity systematically decreased and the WN spectral type became cooler. In 1994, it underwent an LBV-type eruption with a more than 2 magnitude visual brightness increase, a significant decrease in wind velocity, and a spectral type of WN11 or B1.5 $\mathrm{Ia}^{+}$. Within a few months after light curve maximum, the fast wind resumed at the same time that the spectrum reverted to a hotter WN type (WN6 or 7). A summary of the characteristics of the system and additional references can be found in Barbá et al. (1997), Koenigsberger et al. (1998a; 1998b), Moffat et al. (1998), and Niemela et al. (1997).

The following is a working scenario for HD 5980: There are (at least) two very massive stars (perhaps $85 M_{\odot}+30 M_{\odot}$ ) in a close binary system, one of which (star A) systematically increased its radius and mass-loss rate until its dimensions approached its Roche Lobe. The gradual increase in its radius appears to have occurred over the first few years between 1978 and 1991. Starting in 1991, the radius increase appears to be intermittent, with a first important outburst (implying a large radius increase) ocurring in 1993 (see Breysacher (1997) for the long term light curve behavior). It has not yet been determined whether the mechanism producing the outburst is related 
to evolutionary changes, as in more typical LBV's, or, whether, as proposed by Moreno et al. (1997), the forced oscillations induced by the eccentric orbit have led to the instability. However, the fact remains that star A's radius and mass loss rate grew significantly, and the wind velocity diminished throughout this process. At the time of the eruption, the mass loss rate of star $\mathrm{A}$ is $\sim 5$ $\times 10^{-4} M_{\odot} \mathrm{yr}^{-1}$, and its spectrum presents lines arising from a wide range of ionization potentials, with a large variety of wind velocities deduced from the $P$ Cygni profiles in the UV lines. Their velocity components go from near $200 \mathrm{~km} \mathrm{~s}^{-1}$ for the low ionization lines up to $1700 \mathrm{~km} \mathrm{~s}^{-1}$ for Si IV and C IV.

In Figure 2 we illustrate the Si IV 1393 and $1402 \AA$ line profiles in IUE spectra obtained at two different epochs: near maximum in the eruption (SWP52888; obtained in 1994 November) and 250 days later (SWP 55394). Both spectra are at the same orbital phase (phase $=0.50)$, corresponding to an orbital position very near apoastron. The Si IV line profiles in SWP 52888 have the following peculiar features: 1) Despite being resonance lines, they do not saturate; i.e., the minimum intensity of the P Cygni absorption component does not go down to zero flux; 2) there are several sharp emission features superimposed upon the absorption component, the most prominent of which are at $-900,-1200$, and $-1480 \mathrm{~km} \mathrm{~s}^{-1}$ (Koenigsberger et al. 1995); 3) the absorption component of the Si IV 1393 line extends out to $-1700 \mathrm{~km}$ $\mathrm{s}^{-1}$ (the same as C IV 1550), while most of the other lines display maximum velocities slower than $800 \mathrm{~km} \mathrm{~s}^{-1}$. Although orbital phase-dependent line profile changes are generally present, there are many features in the spectrum at the time of the 1994 outburst which remain stable with orbital phase. This implies that the line forming region extends way beyond the orbital separation.

The fact that the Si IV absorption components do not reach zero flux intensity implies that either the region containing these ions does not cover entirely the stellar continuum emission and/or these absorptions are "filled

in" by the superimposed narrow emission components. This suggests that Si IV ions could be concentrated to the equatorial plane of the binary, with the morphology of a disk which is thinner than the radius of the erupting star. It is interesting to note that the signature of this possible disk is still present 250 days later, as can be seen in the narrow absorption components at $-850 \mathrm{~km} \mathrm{~s}^{-1}$ in SWP 55394 .

\section{Conclusions}

The analysis of particle trajectories in a massive binary system in which one of the two stars has a slow wind indicates that a significant fraction of the wind particles are deflected towards the orbital plane. This suggests that in the LBV phase, a circumbinary disk-like structure can be formed as a consequence of the diminution in the stellar wind velocity, the gravitational force 


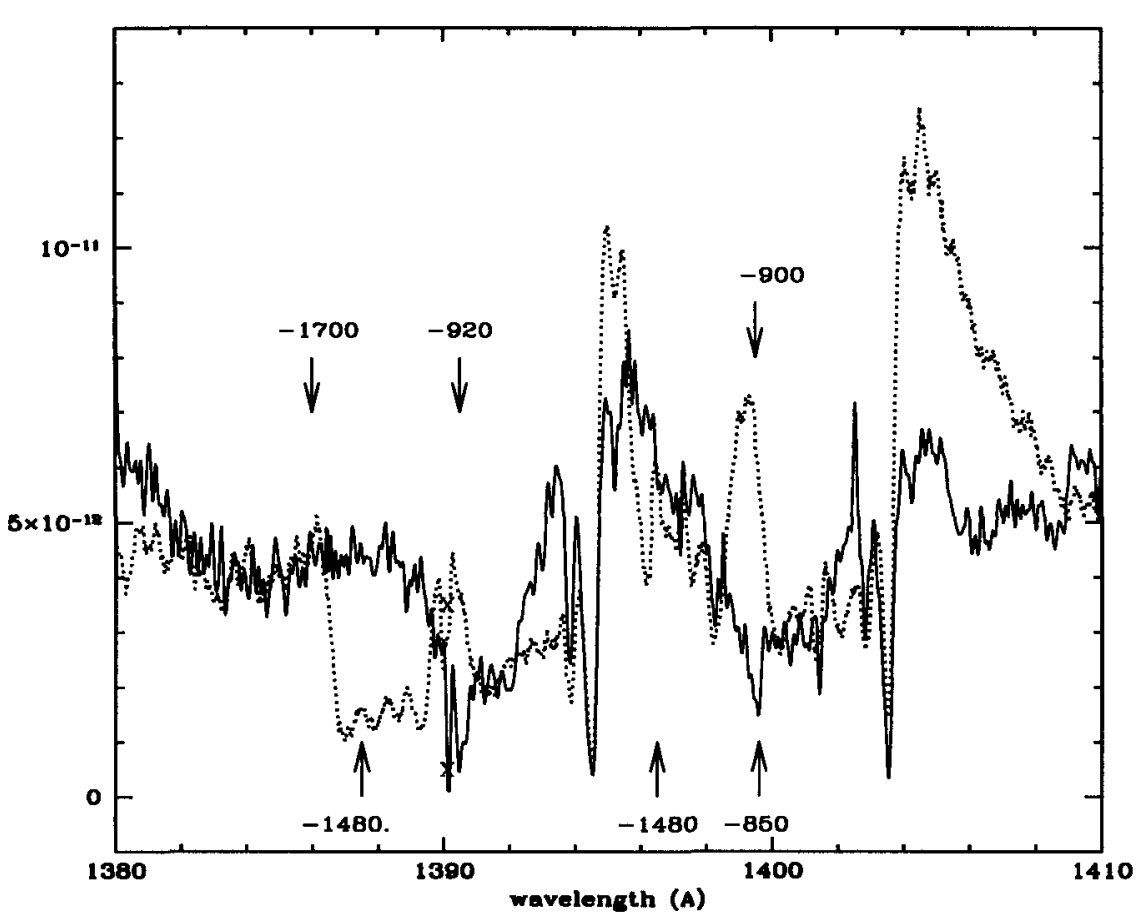

Fig. 2. IUE line profiles of the Si IV 1393, $1402 \AA$ doublet in HD 5980: SWP 52888 (dotted tracing) and SWP 55394. The ordinate is un-dereddened flux in units of ergs $\mathrm{cm}^{-2} \mathrm{~s}^{-1} \AA^{-1}$. Velocities of various features, in $\mathrm{km} \mathrm{s}^{-1}$ and corrected for the SMC motion of $+150 \mathrm{~km} \mathrm{~s}^{-1}$, are indicated." $\mathrm{X}$ " indicates a Reseau fiducial point.

of the companion, and the orbital motion. A more detailed analysis, including the effects of radiation pressure and the collision of particles, is required in order to describe more precisely the characteristics of such a circumbinary structure. However, it is important to point out that the preliminary results of this model suggest that the ionization and velocity structures in the wind at large distances from the star in a massive binary system are not smooth, and that multiple shock regions are expected. Some of the UV spectral characteristics observed in the LBV/WR system HD 5980 are consistent with the idea of a circumbinary structure, although the effects of the companion's wind and radiation field need to be incorporated. Finally, it should be noted that the morphology of the wind-wind collision region between the two stars 
may change significantly from that predicted by stationary-flow models, a circumstance which should be analyzed in the future.

Acknowledgements. We thank Jon Bjorkman for discussing with us the expected effects on the UV line profiles due to the presence of a disk in HD 5980. This work was supported in part by UNAM/DGAPA grants. Juana Orta is gratefully acknowledged for her help in preparing the text.

\section{References}

Barbá, R., Niemela, V., Morel, N. (1997): in Luminous Blue Variables: Massive Stars in Transition, ASP Conf. Ser. 120, 238.

Bodenheimer, P., Tamm, R.E. (1984): ApJ, 280, 771

Breysacher, J. (1997): in Luminous Blue Variables: Massive Stars in Transition, ASP Conf. Ser. 120, 227.

Cassinelli, J., Ignace, R., Bjorkman, J. (1995) in IAU Symposium 163, WR Stars: Binaries, Colliding Winds, Evolution, eds. K.A. van der Hucht and P.M. Williams, (Dordrecht:Kluwer), p.191.

Damineli, A., Conti, P., Lopes, D.F., 1997, NewA 2, 107.

Drissen, L., Leitherer, C., Nota, A. (1992): Nonisotropic and Variable Outflows from Stars, ASP Conf. Ser. 22.

Fliegner, J., Langer, N. (1995), in IAU Symposium 163, WR Stars: Binaries, Colliding Winds, Evolution, eds. K.A. van der Hucht and P.M. Williams, (Dordrecht:Kluwer), p.326.

Frank, A. (1997): in Luminous blue Variables: massive stars in Transition, ASP Conf. Ser. $120,338$.

Garcia-Segura, G., Langer, N., MacLow, M.M. (1997): in Luminous blue Variables: massive stars in Transition, ASP Conf. Ser. 120, 332.

Iben, I., Livio, M. (1993): ASP, 105, 1373.

Koenigsberger, G., Guinan, E., Auer, L. H., Georgiev, G. , (1995): ApJ, 452, L.107.

Koenigsberger, G., Auer, L. H., Georgiev, L., Guinan, E. (1998a):ApJ, 496, 934.

Koenigsberger, G., Peña, M., Schmutz, W., Ayala, S. (1998b): ApJ, 499, 889.

Lamers, H.J.G.L.M., Livio, M., Panagia, N., Walborn, N., (1998), ApJ in press.

Livio, M., Soker, N. (1988): ApJ, 329, 764.

Moffat, A.F.J., Marchenko, S., Bartzakos, P., Niemela, V., Cerruti, M.A., Magalhaes, A.M., Balona, L., St.-Louis, N., Seggewiss, W., Lamontagne, R. (1998) ApJ in press.

Moreno, E., Georgiev, L., Koenigsberger, G. (1997): in Luminous Blue Variables: Massive Stars in Transition, ASP Conf. Ser. 120, 152.

Niemela, Barbá, R., Morrell, N., Corti, M., (1997): in Luminous Blue Variables: Massive Stars in Transition, ASP Conf. Ser. 120,

Taam, R.E., Bodenheimer, P. (1989): ApJ, 337, 849

Taam, R.E., Bodenheimer, P. (1991): ApJ, 373, 246.

Terman, J.L., Taam, R.E., Hernquist, L. (1994), ApJ, 422, 729.

White, $\dot{R}$. L., Becker, R.H. (1995), ApJ, 451, 352. 


\section{Discussion}

J. Echevarria: Do you see any spectroscopic evidence for a disk during or after the LBV eruption event of 1994 ?

G. Koenigsberger: The Si IV $\lambda 1393$ line profile is suggestive of an outflowing disk, but modelling is required before a conclusion can be drawn.

$H$. Henrichs: Have you also analysed the Si III $\lambda 1206$ line behaviour? Your spectra looked promising.

G. Koenigsberger: I have not, but will do so as soon as possible.

K. van der Hucht: Do you have a series of consecutive IUE observations, let's say 10 or 20 within one 13-day binary period, to separate the variations within one orbital period from long-term variations?

G. Koenigsberger: No, unfortunately at most only a few observing shifts within one orbital period were possible.

M. Friedjung: The mass-loss rate during the eruption is very large and I would expect the wind to be optically thick in the continuum. I presume this mass loss must only be in the orbital plane to avoid occultations.

R. Schulte-Ladbeck: If you are interested in the presence of a circumbinary disk, you might be able to detect it with broad-band polarisation monitoring: analyse the Fourier components in the time series (cf. Brown et al.).

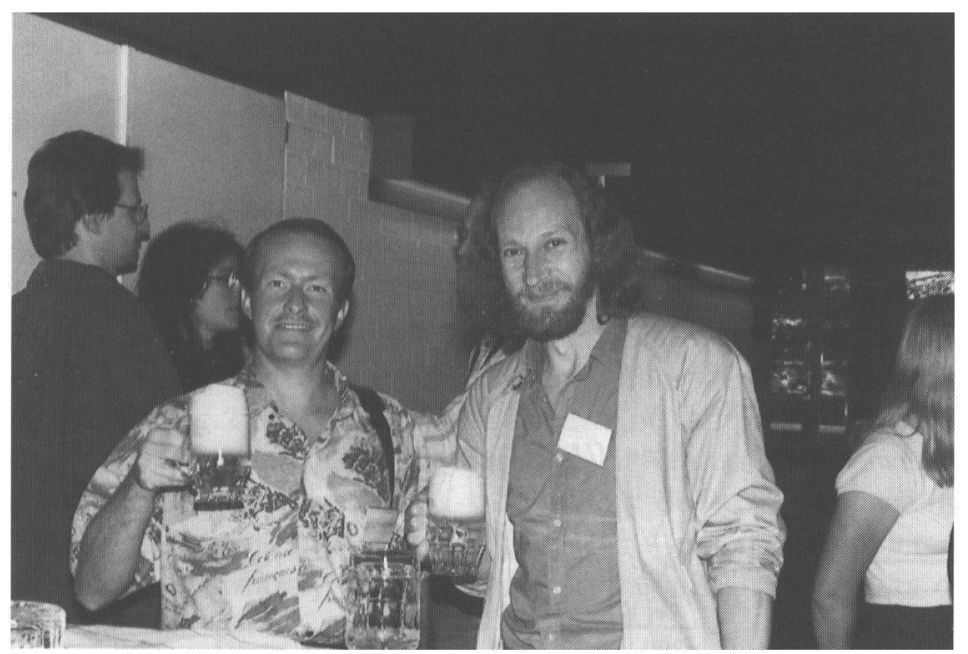

Anatoly Miroshnichenko and Ruslan Yudin 